\title{
Linear Motion Observers for ASC/AUV Tandems based on Single Range Readings
}

\author{
D.Viegas, P. Batista, P. Oliveira, and C. Silvestre
}

\begin{abstract}
This paper proposes novel cooperative navigation strategies for an Intervention Autonomous Underwater Vehicle (I-AUV) working in tandem with an Autonomous Surface Craft (ASC). The proposed solutions rely on single range readings and two different scenarios are considered: in the first, the I-AUV is assumed to be moving in the presence of unknown constant ocean currents and the ASC transmits its inertial position and velocity, while the I-AUV measures its velocity relative to the water; in the second case, the ASC transmits its inertial position and the I-AUV is assumed to measure its velocity relative to the ASC. Necessary and sufficient observability conditions are derived for both problems and globally asymptotically stable (GAS) filters are developed. Although inspired in marine robotics, the proposed methods apply to other mobile platforms as they are based on the general motion kinematics in 3-D. Finally, to assess the performance of the proposed solutions, realistic simulation results are presented and discussed.
\end{abstract}

\section{INTRODUCTION}

Nowadays, an increasing number of field operations with unmanned underwater vehicles (UUVs) in applications like marine rescue, marine science, and the offshore industries, to mention only but a few, require intervention capabilities in order to perform the desired tasks. While the technology to perform such tasks is already available, the level of autonomy and perception required for autonomous intervention has not yet been attained and current operations are mostly undertaken by manned submersibles or by Remotely Operated Vehicles (ROVs), both equipped with the required tools to perform the appropriate interventions. Although successful, these options pose strong drawbacks and liabilities. Manned submersibles have reduced operation times, in general, and require human presence in a dangerous and hostile environment, which is certainly undesirable, in addition to the high costs associated with the support oceanographic vessel. ROVs require, in addition to a support vessel, an automatic Tether Management System (TMS) and a Dynamic Position (DP) system. Moreover, their operation time is limited by the fatigue of the pilot and other operators. These issues, among others, have motivated the research community to promote new solutions, in particular the development of Intervention Autonomous Underwater Vehicles (I-AUV). In this context, project TRIDENT, funded by the European Union, aims to develop a team of two cooperative heterogeneous robots with complementary skills, an Autonomous Surface Craft (ASC) and an I-AUV, endowed with a dexterous manipulator, which

This work was partially funded by Fundao para a Cincia e a Tecnologia (ISR/IST plurianual funding) through the PIDDAC Program funds and EU Project TRIDENT (Contract No. 248497).

The authors are with the Institute for Systems and Robotics, Instituto Superior Técnico, Av. Rovisco Pais, 1049-001 Lisboa, Portugal. $\{d v i e g a s$, pbatista, pjcro, cjs\}@isr.ist.utl.pt will be used to perform underwater manipulation tasks. This paper proposes a novel navigation strategy for the I-AUV based on single range readings from the I-AUV to the ASC, in addition to auxiliary sensors to drive the system dynamics and cooperative communication between the two vehicles.

While the design of navigation systems for ASCs is fairly well understood, see e.g. [1] for an experimentally validated navigation solution for the DELFIMx, an ASC associated with project TRIDENT, the problem of underwater navigation is still a very active field of research, see [2] for a recent survey on this topic. Dead Reckoning (DR) navigation provides very good short term results but its performance necessarily degrades over time, see [3]. Artificial beacons may be employed for long term underwater positioning but, even though there exist many different configurations such as Long Base Line (LBL), Short Base Line (SBL) or Ultra Short Baseline (USBL) [4], [5], [6], operational constraints render these solutions unfeasible for numerous applications, as there exists the need of prior beacon deployment and accurate position assertion. More recently, cooperative navigation solutions based on range measurements have been studied in [7], [8], and [9]. This paper provides an alternative solution, where the I-AUV designs a navigation system with respect to the ASC, which also communicates with the I-AUV for appropriate geo-referencing.

This paper addresses the problem of cooperative navigation/source localization based on range measurements to a single mobile source, aided by transmission of relevant data from the source to the agent, in the presence of unknown constant drifts. Two scenarios are considered: in the first, the source transmits its inertial position and velocity to the agent, while in the second case it only sends its inertial position. In addition to the range readings, the agent is assumed to measure the velocity relative to the fluid, in the first case, or the velocity relative to the source, in the second case. In the particular case of an ASC and an I-AUV working in tandem, a Doppler Velocity Log (DVL) provides the velocity of the I-AUV relative to the water, while an Acoustic VectorSensor Array [10] gives the velocity of the I-AUV relative to the ASC. For both cases, the nonlinear systems are analyzed and necessary and sufficient conditions are derived for their observability. The observability analysis is driven by the derivation through state augmentation of two Linear TimeVarying (LTV) systems that mimic exactly the dynamics of the nonlinear systems. While this paper is motivated by the practical case of an I-AUV working in tandem with an ASC, the dynamic systems used to model the problem are built on the linear motion kinematics of agents moving in 3-D. As such, the results can be extrapolated to other 
scenarios involving different types of vehicles, therefore broadening the usefulness of the present work. Finally, as the observability analysis resulted in a constructive process, a Kalman filter with globally asymptotically stable error dynamics is also proposed. Previous work by the authors on the subject of linear motion estimation based on range measurements can be found in [11], where the case with a stationary source was considered. This paper extends those results and also provides enhanced proofs.

The paper is organized as follows: Section II introduces the problem framework and presents motivation examples, while Section III details the observability analysis. Simulation results are shown in Section IV to evaluate the proposed solution and, finally, Section V summarizes the main conclusions of the paper.

\section{A. Notation}

Throughout the paper the symbol 0 denotes a matrix (or vector) of zeros and I an identity matrix, both of appropriate dimensions. A block diagonal matrix is represented as $\operatorname{diag}\left(\mathbf{A}_{1}, \ldots, \mathbf{A}_{\mathbf{n}}\right)$. For $\mathbf{x}, \mathbf{y} \in \mathbb{R}^{3}, \mathbf{x} \times \mathbf{y}$ represents the cross product.

\section{Problem Statement}

Consider a point-mass agent moving in three dimensions and suppose that the agent has always access to measurements of its distance, or range, $r(t)$ to a mobile source. The problem of mobile source localization by mobile agents considered in the paper is that of estimating the position of the source from the knowledge of $r(t)$. To complete the problem framework, let $\{I\}$ denote an inertial reference coordinate frame and $\{B\}$ a coordinate frame attached to the agent, denominated in the sequel as the body-fixed coordinate frame. The linear motion of the agent can be written as

$$
\dot{\mathbf{p}}(t)=\mathbf{R}(t) \mathbf{v}(t),
$$

where $\mathbf{p}(t) \in \mathbb{R}^{3}$ denotes the inertial position of the agent, $\mathbf{v}(t) \in \mathbb{R}^{3}$ is the velocity of the agent relative to $\{I\}$ and expressed in body-fixed coordinates, and $\mathbf{R}(t)$ is the rotation matrix from $\{B\}$ to $\{I\}$, which satisfies

$$
\dot{\mathbf{R}}(t)=\mathbf{R}(t) \mathbf{S}(\boldsymbol{\omega}(t)),
$$

where $\boldsymbol{\omega}(t) \in \mathbb{R}^{3}$ is the angular velocity of $\{B\}$, expressed in body-fixed coordinates, and $\mathbf{S}(\boldsymbol{\omega}(t))$ is the skew-symmetric matrix such that $\mathbf{S}(\boldsymbol{\omega}(t)) \boldsymbol{x}$ is the cross product $\boldsymbol{\omega} \times \boldsymbol{x}$. Let $\mathbf{s}(t) \in \mathbb{R}^{3}$ denote the inertial position of the source, and $\mathbf{v}_{s}(t) \in \mathbb{R}^{3}$ its inertial velocity. Then, the range to the source is given by $r(t)=\|\mathbf{r}(t)\|$, where

$$
\mathbf{r}(t):=\mathbf{R}^{T}(t)[\mathbf{s}(t)-\mathbf{p}(t)]
$$

is the position of the source relative to the agent, expressed in body-fixed coordinates, precisely the quantity that the agent aims to estimate. The time derivative of (1) is given by

$$
\dot{\mathbf{r}}(t)=-\mathbf{S}(\boldsymbol{\omega}(t)) \mathbf{r}(t)+\mathbf{R}^{T}(t) \mathbf{v}_{s}(t)-\mathbf{v}(t) .
$$

Consider the case of an I-AUV moving underwater in the presence of constant unknown ocean currents and working cooperatively with an ASC that takes the role of the mobile source. It is assumed that the ASC has a built-in navigation system which provides accurate estimates of both its inertial position and velocity, and can communicate them to the I-AUV (using, e.g., an accoustic modem). The agent can then recover its own inertial position by comparing $\mathbf{r}(t)$ with the position of the ASC. It is further assumed that the sensor suite on-board the I-AUV provides measurements of its attitude and angular velocity, $\mathbf{R}(t)$ and $\boldsymbol{\omega}(t)$. As the agent is evolving in an environment where unknown currents are present, it might not be possible to measure its inertial velocity directly, for example if the I-AUV is moving far away from the seabed. However, its velocity relative to the fluid is available, as measured by a DVL. Regarding the velocity of the source, two cases will be considered. In the first case, the source communicates its velocity, $\mathbf{v}_{s}(t)$, to the agent, and in the second case, the I-AUV has access to measurements of its velocity relative to the source,

$$
\Delta \mathbf{v}(t)=\mathbf{R}^{T}(t) \mathbf{v}_{s}(t)-\mathbf{v}(t),
$$

provided by the Acoustic Vector-Sensor Array.

Let $\mathbf{v}_{r}(t) \in \mathbb{R}^{3}$ and $\mathbf{v}_{f}(t) \in \mathbb{R}^{3}$ denote the velocity of the agent relative to the fluid and the velocity of the fluid relative to $\{I\}$, respectively, both expressed in bodyfixed coordinates. Considering that the velocity of the fluid is constant, it is possible to further write

$$
\left\{\begin{array}{l}
\dot{\mathbf{r}}(t)=-\mathbf{S}(\boldsymbol{\omega}(t)) \mathbf{r}(t)+\mathbf{R}^{T}(t) \mathbf{v}_{s}(t)-\mathbf{v}_{r}(t)-\mathbf{v}_{f}(t) \\
\dot{\mathbf{v}}_{f}(t)=-\mathbf{S}(\boldsymbol{\omega}(t)) \mathbf{v}_{f}(t)
\end{array} .\right.
$$

Now, let

$$
\left\{\begin{array}{l}
{\left[\begin{array}{l}
\mathbf{x}_{1}(t) \\
\mathbf{x}_{2}(t)
\end{array}\right]=\left[\begin{array}{cc}
\mathbf{R}(t) & \mathbf{0} \\
\mathbf{0} & -\mathbf{R}(t)
\end{array}\right]\left[\begin{array}{c}
\mathbf{r}(t) \\
\mathbf{v}_{f}(t)
\end{array}\right],} \\
y(t)=r(t)^{2}=\|\mathbf{r}(t)\|^{2}=\left\|\mathbf{x}_{1}(t)\right\|^{2}
\end{array}\right.
$$

which is a Lyapunov coordinate transformation, and thus preserves stability and observability properties [12], and define

$$
\mathbf{u}(t)=\mathbf{v}_{s}(t)-\mathbf{R}(t) \mathbf{v}_{r}(t) .
$$

Computing the time derivatives of $\mathbf{x}_{1}(t)$ and $\mathbf{x}_{2}(t)$ gives the nonlinear system

$$
\left\{\begin{array}{l}
\dot{\mathbf{x}}_{1}(t)=\mathbf{x}_{2}(t)+\mathbf{u}(t) \\
\dot{\mathbf{x}}_{2}(t)=\mathbf{0} \\
y(t)=\left\|\mathbf{x}_{1}(t)\right\|^{2}
\end{array}\right.
$$

where $\mathbf{x}_{1}(t), \mathbf{x}_{2}(t) \in \mathbb{R}^{3}$ are the system states, $\mathbf{u}(t) \in \mathbb{R}^{3}$ is the system input, and $y(t) \in \mathbb{R}$ represents the system output.

Regarding the second case, the time derivative of $\mathbf{r}(t)$ is reduced to $\dot{\mathbf{r}}(t)=-\mathbf{S}(\boldsymbol{\omega}(t)) \mathbf{r}(t)+\Delta \mathbf{v}(t)$. Now, let

$$
\left\{\begin{array}{l}
\mathbf{x}_{1}(t)=\mathbf{R}(t) \mathbf{r}(t) \\
y(t)=r(t)^{2}=\|\mathbf{r}(t)\|^{2}=\left\|\mathbf{x}_{1}(t)\right\|^{2},
\end{array}\right.
$$

which is also a Lyapunov coordinate transformation, and define $\mathbf{u}(t):=\Delta \mathbf{v}(t)$. Computing the time derivative of $\mathbf{x}_{1}(t)$ gives the nonlinear system

$$
\left\{\begin{array}{l}
\dot{\mathbf{x}}_{1}(t)=\mathbf{u}(t) \\
y(t)=\left\|\mathbf{x}_{1}(t)\right\|^{2}
\end{array},\right.
$$

where $\mathbf{x}_{1}(t) \in \mathbb{R}^{3}$ is the system state, $\mathbf{u}(t) \in \mathbb{R}^{3}$ is the system input, and $y(t) \in \mathbb{R}$ is the system output.

The problems considered in the paper are the observability analysis of the nonlinear systems (2) and (3) and the design of state observers for those systems. 


\section{OBSERVABILITy ANALYSIS}

This section details the observability analysis of (2) and (3) through state augmentation. With the proposed methodologies, it is possible to derive linear systems which capture the behavior of the nonlinear systems, and as such study their observability in a linear systems framework. The observability analysis of (2) is detailed in subsections A through C: Subsection A introduces the augmented system dynamics and Subsection B details a sufficient condition for its observability, while Subsection C establishes necessary and sufficient conditions for observability of the nonlinear system. Subsections D through F refer to the observability analysis of (3) and are organized in a similar fashion.

\section{A. State Augmentation (first case)}

To derive a linear system that mimics the dynamics of the nonlinear system (2), define three additional scalar state variables as

$$
\left\{\begin{array}{l}
x_{3}(t)=y(t) \\
x_{4}(t)=\mathbf{x}_{1}^{T}(t) \mathbf{x}_{2}(t) \\
x_{5}(t)=\left\|\mathbf{x}_{2}(t)\right\|^{2}
\end{array}\right.
$$

and denote by

$$
\mathbf{x}(t)=\left[\mathbf{x}_{1}^{T}(t) \mathbf{x}_{2}^{T}(t) x_{3}(t) x_{4}(t) x_{5}(t)\right]^{T} \in \mathbb{R}^{n}, n=9,
$$

the augmented state. Then, the dynamics of the augmented system can be written as

$$
\left\{\begin{array}{l}
\dot{\mathbf{x}}(t)=\mathbf{A}(t) \mathbf{x}(t)+\mathbf{B u}(t) \\
y(t)=\mathbf{C x}(t)
\end{array}\right.
$$

where

$$
A(t)=\left[\begin{array}{ccccc}
\mathbf{0} & \mathbf{I} & \mathbf{0} & \mathbf{0} & \mathbf{0} \\
\mathbf{0} & \mathbf{0} & \mathbf{0} & \mathbf{0} & \mathbf{0} \\
2 \mathbf{u}^{T}(t) & \mathbf{0} & 0 & 2 & 0 \\
\mathbf{0} & \mathbf{u}^{T}(t) & 0 & 0 & 1 \\
\mathbf{0} & \mathbf{0} & 0 & 0 & 0
\end{array}\right], B=\left[\begin{array}{l}
\mathbf{I} \\
\mathbf{0} \\
0 \\
0 \\
0
\end{array}\right]
$$

and $\mathbf{C}=\left[\begin{array}{lllll}\mathbf{0} & \mathbf{0} & 1 & 0 & 0\end{array}\right]$.

The dynamic system (4) can then be regarded as a linear time-varying system, and its observability will be analyzed using classical linear systems theory, applied to the pair $(\mathbf{A}(t), \mathbf{C})$. Notice that there is nothing in (4) imposing

$$
\left\{\begin{array}{l}
y(t)=\left\|\mathbf{x}_{1}(t)\right\|^{2} \\
x_{4}(t)=\mathbf{x}_{1}^{T}(t) \mathbf{x}_{2}(t) \\
x_{5}(t)=\left\|\mathbf{x}_{2}(t)\right\|^{2}
\end{array}\right.
$$

so the observability of (4) does not automatically entail the observability of (2).

\section{B. Observability of the linear system (first case)}

Before showing a sufficient condition for the observability of (4), it is convenient to compute the transition matrix associated with $\mathbf{A}(t)$. Let

$$
\mathbf{u}^{[1]}\left(t, t_{0}\right):=\int_{t_{0}}^{t} \mathbf{u}(\sigma) d \sigma=\left[\begin{array}{l}
u_{1}^{[1]}\left(t, t_{0}\right) \\
u_{2}^{[1]}\left(t, t_{0}\right) \\
u_{3}^{[1]}\left(t, t_{0}\right)
\end{array}\right] .
$$

Then, the transition matrix can be written as

$$
\left[\begin{array}{ccccc}
\phi\left(t, t_{0}\right)= & & \\
\mathbf{I} & \left(t-t_{0}\right) \mathbf{I} & \mathbf{0} & \mathbf{0} & \mathbf{0} \\
\mathbf{0} & \mathbf{I} & \mathbf{0} & \mathbf{0} & \mathbf{0} \\
2\left[\mathbf{u}^{[1]}\left(t, t_{0}\right)\right]^{T} & 2\left(t-t_{0}\right)\left[\mathbf{u}^{[1]}\left(t, t_{0}\right)\right]^{T} & 1 & 2\left(t-t_{0}\right) & \left(t-t_{0}\right)^{2} \\
\mathbf{0} & {\left[\mathbf{u}^{[1]}\left(t, t_{0}\right)\right]^{T}} & 0 & 1 & t-t_{0} \\
\mathbf{0} & \mathbf{0} & 0 & 0 & 1
\end{array}\right] .
$$

The observability Gramian for the pair $(\mathbf{A}(t), \mathbf{C})$ is given by

$$
\mathcal{W}\left(t_{0}, t_{f}\right)=\int_{t_{0}}^{t_{f}} \phi^{T}\left(t, t_{0}\right) \mathbf{C}^{T} \mathbf{C} \phi\left(t, t_{0}\right) d t .
$$

Note that, due to the specific structure of $\mathbf{C}$, the above expression can be reduced to

$$
\mathcal{W}\left(t_{0}, t_{f}\right)=\int_{t_{0}}^{t_{f}} \phi_{3}^{T}\left(t, t_{0}\right) \phi_{3}\left(t, t_{0}\right) d t
$$

where $\phi_{3}\left(t, t_{0}\right)$ is the line of the transition matrix (5) associated with $x_{3}$. The following result presents a sufficient condition for the system (4) to be observable.

Theorem 1: Suppose that the following set of functions

$$
\begin{aligned}
\mathcal{F}=\{ & \left(t-t_{0}\right),\left(t-t_{0}\right)^{2}, u_{1}^{[1]}\left(t, t_{0}\right), u_{2}^{[1]}\left(t, t_{0}\right), u_{3}^{[1]}\left(t, t_{0}\right), \\
& \left.\left(t-t_{0}\right) u_{1}^{[1]}\left(t, t_{0}\right),\left(t-t_{0}\right) u_{2}^{[1]}\left(t, t_{0}\right),\left(t-t_{0}\right) u_{3}^{[1]}\left(t, t_{0}\right)\right\}
\end{aligned}
$$

is linearly independent on $\left[t_{0}, t_{f}\right], t_{0}<t_{f}$. Then, the LTV system (5) is observable on $\left[t_{0}, t_{f}\right]$ in the sense that, given the system input $\mathbf{u}(t), t \in\left[t_{0}, t_{f}\right]$, and the system output $y(t), t \in\left[t_{0}, t_{f}\right]$, the initial condition is uniquely defined.

Proof: Suppose that the LTV system (4) is not observable on $\left[t_{0}, t_{f}\right]$. Then, the observability Gramian $\mathcal{W}\left(t_{0}, t_{f}\right)$ is not positive definite and therefore

$$
\begin{aligned}
& \underset{\mathbf{C}^{n}}{\exists} \quad \forall \quad: \mathbf{d}^{T} \mathcal{W}\left(t_{0}, t_{f}\right) \mathbf{d}=0 . \\
& \|\mathbf{d}\|=1
\end{aligned}
$$

Let $\mathbf{d}=\left[\begin{array}{lllll}\mathbf{d}_{1}^{T} & \mathbf{d}_{2}^{T} & d_{3} & d_{4} & d_{5}\end{array}\right] \in \mathbb{R}^{n}$, where $\mathbf{d}_{1}, \mathbf{d}_{2} \in$ $\mathbb{R}^{3}$. Expanding (8) and using (6) gives

$$
\int_{t_{0}}^{t}\left[\phi_{3}\left(\sigma, t_{0}\right) \mathbf{d}\right]^{2} d \sigma=0
$$

for all $t \in\left[t_{0}, t_{f}\right]$, and it follows that

$$
\phi_{3}\left(t, t_{0}\right) \mathbf{d}=0
$$

for all $t \in\left[t_{0}, t_{f}\right]$. But $\phi_{3}\left(t_{0}, t_{0}\right) \mathbf{d}=d_{3}$, so for (9) to hold, it must be $d_{3}=0$. From (9), it also follows that

$$
\frac{d}{d t} \phi_{3}\left(t, t_{0}\right) \mathbf{d}=0
$$

for all $t \in\left[t_{0}, t_{f}\right]$, yielding

$$
\begin{aligned}
0= & \mathbf{u}^{T}(t) \mathbf{d}_{1}+\left[\mathbf{u}^{[1]}\left(t, t_{0}\right)+\left(t-t_{0}\right) \mathbf{u}(t)\right]^{T} \mathbf{d}_{2} \\
& +d_{4}+\left(t-t_{0}\right) d_{5}
\end{aligned}
$$


for all $t \in\left[t_{0}, t_{f}\right]$. Integrating both sides of (10) gives

$$
\begin{aligned}
0= & {\left[\mathbf{u}^{[1]}\left(t, t_{0}\right)\right]^{T} \mathbf{d}_{1}+\left(t-t_{0}\right)\left[\mathbf{u}^{[1]}\left(t, t_{0}\right)\right]^{T} \mathbf{d}_{2} } \\
& +\left(t-t_{0}\right) d_{4}+\frac{\left(t-t_{0}\right)^{2}}{2} d_{5},
\end{aligned}
$$

which implies that the set of functions $\mathcal{F}$ is not linearly independent on $\left[t_{0}, t_{f}\right]$. Then, if the set of functions $\mathcal{F}$ is linearly independent on $\left[t_{0}, t_{f}\right]$, the observability Gramian must be positive definite, and therefore (4) is observable.

\section{Observability of the Nonlinear System (first case)}

The following theorem provides a necessary condition on the observability of the nonlinear system (2).

Theorem 2: If the nonlinear system (2) is observable on $\left[t_{0}, t_{f}\right], t_{0}<t_{f}$, then

$$
\begin{aligned}
& \nexists \quad \forall \quad: \mathbf{u}_{0}^{T} \mathbf{u}(t)=c . \\
& \mathbf{u}_{0} \in \mathbb{R}^{3} \quad t \in\left[t_{0}, t_{f}\right] \\
& \left\|\mathbf{u}_{0}\right\|=1 \\
& c \in \mathbb{R}
\end{aligned}
$$

Proof: Suppose that (11) does not hold. Then,

$$
\begin{aligned}
& \quad \forall \quad \forall \quad: \mathbf{u}_{0}^{T} \mathbf{u}(t)=c . \\
& \mathbf{u}_{0} \in \mathbb{R}^{3} \quad t \in\left[t_{0}, t_{f}\right] \\
& \left\|\mathbf{u}_{0}\right\|=1 \\
& c \in \mathbb{R}
\end{aligned}
$$

Then, it follows that

$$
\mathbf{u}_{0}^{T} \mathbf{u}^{[1]}\left(t, t_{0}\right)=\mathbf{u}_{0}^{T} \int_{t_{0}}^{t} \mathbf{u}(\sigma) d \sigma=\int_{t_{0}}^{t} \mathbf{u}_{0}^{T} \mathbf{u}(\sigma) d \sigma
$$

and

$$
\mathbf{u}_{0}^{T} \mathbf{u}^{[1]}\left(t, t_{0}\right)=c\left(t-t_{0}\right), \forall t \in\left[t_{0}, t_{f}\right] .
$$

The output of the system (2) is given by

$y(t)=\left\|\mathbf{x}_{1}(t)\right\|^{2}=\left\|\mathbf{x}_{1}\left(t_{0}\right)+\left(t-t_{0}\right) \mathbf{x}_{2}\left(t_{0}\right)+\int_{t_{0}}^{t} \mathbf{u}(\sigma) d \sigma\right\|^{2}$.

Let $y^{a}(t)$ denote the output of the system with initial condition

$$
\left\{\begin{array}{l}
\mathbf{x}_{1}^{a}\left(t_{0}\right)=\mathbf{u}_{0} \\
\mathbf{x}_{2}^{a}\left(t_{0}\right)=-c \mathbf{u}_{0}
\end{array}\right.
$$

and $y^{b}(t)$ denote the output of the system with initial condition

$$
\left\{\begin{array}{l}
\mathbf{x}_{1}^{b}\left(t_{0}\right)=-\mathbf{u}_{0} \\
\mathbf{x}_{2}^{b}\left(t_{0}\right)=-c \mathbf{u}_{0}
\end{array} .\right.
$$

The output of the system with the first set of initial conditions is

$$
\begin{aligned}
y^{a}(t) & =\left\|x_{1}^{a}\left(t_{0}\right)+\left(t-t_{0}\right) \mathbf{x}_{2}^{a}\left(t_{0}\right)+\int_{t_{0}}^{t} \mathbf{u}(\sigma) d \sigma\right\|^{2} \\
& =1+c^{2}\left(t-t_{0}\right)^{2}+\left\|\mathbf{u}^{[1]}\left(t, t_{0}\right)\right\|^{2}-2 c^{2}\left(t-t_{0}\right)^{2},
\end{aligned}
$$

while its output with the second set of initial conditions is

$$
\begin{aligned}
y^{b}(t) & =\left\|x_{1}^{b}\left(t_{0}\right)+\left(t-t_{0}\right) \mathbf{x}_{2}^{b}\left(t_{0}\right)+\int_{t_{0}}^{t} \mathbf{u}(\sigma) d \sigma\right\|^{2} \\
& =1+c^{2}\left(t-t_{0}\right)^{2}+\left\|\mathbf{u}^{[1]}\left(t, t_{0}\right)\right\|^{2}-2 c^{2}\left(t-t_{0}\right)^{2}
\end{aligned}
$$

which means that $y^{a}(t)=y^{b}(t), \forall t \in\left[t_{0}, t_{f}\right]$. Thus, if (11) does not hold, there exist, at least, two different states that are indistinguishable, and therefore the system is not observable, which concludes the proof.

The following theorem provides a sufficient condition for the observability of the nonlinear system (2), as well as a practical result that can be used in the design of state observers for that system.

Theorem 3: Suppose that the set of functions (7) is linearly independent on $\left[t_{0}, t_{f}\right], t_{0}<t_{f}$. Then, the nonlinear system (2) is observable on $\left[t_{0}, t_{f}\right]$ in the sense that, given the system input $\mathbf{u}(t), t \in\left[t_{0}, t_{f}\right]$, and the system output $y(t), t \in\left[t_{0}, t_{f}\right]$, the initial condition is uniquely defined. Moreover, a state observer with globally asymptotically stable error dynamics for the LTVS (4) is also a state observer for the nonlinear system (2), with globally asymptotically stable error dynamics.

Proof: Let $\left.\left[\mathbf{x}_{1}^{T}\left(t_{0}\right) x_{2}^{T}\left(t_{0}\right)\right]^{T}\right]$ be the initial state of the nonlinear system (2). Then, the output is given by

$$
\begin{aligned}
y(t)= & \left\|\mathbf{x}_{1}\left(t_{0}\right)+\left(t-t_{0}\right) \mathbf{x}_{2}\left(t_{0}\right)+\int_{t_{0}}^{t} \mathbf{u}(\sigma) d \sigma\right\|^{2} \\
= & \left\|\mathbf{x}_{1}\left(t_{0}\right)\right\|^{2}+\left\|\mathbf{x}_{2}\left(t_{0}\right)\right\|^{2}\left(t-t_{0}\right)^{2}+\left\|\mathbf{u}^{[1]}\left(t, t_{0}\right)\right\|^{2} \\
& +2\left(t-t_{0}\right) \mathbf{x}_{1}^{T}\left(t_{0}\right) \mathbf{x}_{2}\left(t_{0}\right)+2 \mathbf{x}_{1}^{T}\left(t_{0}\right) \mathbf{u}^{[1]}\left(t, t_{0}\right) \\
& +2\left(t-t_{0}\right) \mathbf{x}_{2}^{T}\left(t_{0}\right) \mathbf{u}^{[1]}\left(t, t_{0}\right) .
\end{aligned}
$$

Since the set of functions (7) is assumed linearly independent on $\left[t_{0}, t_{f}\right]$ it follows, from Theorem 2 , that the LTVS (4) is observable on $\left[t_{0}, t_{f}\right]$. Thus, given $y(t)$ for $t \in\left[t_{0}, t_{f}\right]$, the initial state of (4) is determined uniquely. Let $\left[\mathbf{z}_{1}^{T}\left(t_{0}\right) \mathbf{z}_{2}^{T}\left(t_{0}\right) z_{3}\left(t_{0}\right) z_{4}\left(t_{0}\right) z_{5}\left(t_{0}\right)\right]^{T}$ be the initial state of the linear system (4). Then, the output satisfies

$$
\begin{aligned}
y(t)= & z_{3}(t) \\
= & 2 \mathbf{z}_{1}^{T}\left(t_{0}\right) \mathbf{u}^{[1]}\left(t, t_{0}\right)+2\left(t-t_{0}\right) \mathbf{z}_{2}^{T}\left(t_{0}\right) \mathbf{u}^{[1]}\left(t, t_{0}\right) \\
& +z_{3}\left(t_{0}\right)+2 z_{4}\left(t_{0}\right)\left(t-t_{0}\right)+z_{5}\left(t_{0}\right)\left(t-t_{0}\right)^{2} \\
& +\left\|\mathbf{u}^{[1]}\left(t, t_{0}\right)\right\|^{2} .
\end{aligned}
$$

Notice that $y\left(t_{0}\right)=z_{3}\left(t_{0}\right)=\left\|\mathbf{x}_{1}\left(t_{0}\right)\right\|^{2}$. From the comparison between (12) and (13) it follows that

$$
\begin{aligned}
0= & {\left[\left\|x_{2}\left(t_{0}\right)\right\|^{2}-z_{5}\left(t_{0}\right]\left(t-t_{0}\right)^{2}\right.} \\
& +2\left(t-t_{0}\right)\left[\mathbf{x}_{1}^{T}\left(t_{0}\right) \mathbf{x}_{2}\left(t_{0}\right)-z_{4}\left(t_{0}\right)\right] \\
& +2\left[\mathbf{x}_{1}^{T}\left(t_{0}\right)-\mathbf{z}_{1}^{T}\left(t_{0}\right)\right] \mathbf{u}^{[1]}\left(t, t_{0}\right) \\
& +2\left(t-t_{0}\right)\left[\mathbf{x}_{2}^{T}\left(t_{0}\right)-\mathbf{z}_{2}^{T}\left(t_{0}\right)\right] \mathbf{u}^{[1]}\left(t, t_{0}\right)
\end{aligned}
$$

for all $t \in\left[t_{0}, t_{f}\right]$. Since the set of functions $\mathcal{F}$ is assumed linearly independent, (14) implies that

$$
\left\{\begin{array}{l}
\mathbf{x}_{1}\left(t_{0}\right)=\mathbf{z}_{1}\left(t_{0}\right) \\
\mathbf{x}_{2}\left(t_{0}\right)=\mathbf{z}_{2}\left(t_{0}\right) \\
\mathbf{x}_{1}^{T}\left(t_{0}\right) \mathbf{x}_{2}\left(t_{0}\right)=z_{4}\left(t_{0}\right) \\
\left\|\mathbf{x}_{2}\left(t_{0}\right)\right\|^{2}=z_{5}\left(t_{0}\right)
\end{array} .\right.
$$

This concludes the proof, as both the initial state of the nonlinear system (2) is uniquely determined and the initial state of the linear system (4) matches the initial state of the nonlinear system. 


\section{State Augmentation (second case)}

This subsection and the following establish results for the nonlinear system (3) similar to those shown in previous subsections. In this case, the system is simpler, and therefore only one additional scalar state variable is needed, $x_{2}(t)=$ $y(t)$, and the augmented state is

$$
\mathbf{x}(t)=\left[\begin{array}{ll}
\mathbf{x}_{1}^{T}(t) & x_{2}(t)
\end{array}\right]^{T} \in \mathbb{R}^{n}, n=4 .
$$

The dynamics of the augmented system can be written in the form

$$
\left\{\begin{array}{l}
\dot{\mathbf{x}}(t)=\mathbf{A}_{2}(t) \mathbf{x}(t)+\mathbf{B}_{2} \mathbf{u}(t) \\
y(t)=\mathbf{C}_{2} \mathbf{x}(t)
\end{array},\right.
$$

where

$$
\mathbf{A}_{2}(t)=\left[\begin{array}{cc}
\mathbf{0} & 0 \\
2 \mathbf{u}^{T}(t) & 0
\end{array}\right], \mathbf{B}_{2}=\left[\begin{array}{l}
\mathbf{I} \\
0
\end{array}\right], \text { and } \mathbf{C}_{2}=\left[\begin{array}{ll}
\mathbf{0} & 1
\end{array}\right] .
$$

Similarly to the previous case, this system can be regarded as a linear time-varying system, and its observability will be analyzed in a similar way.

Remark: The nonlinear system (3) is a special case of (2), with $\mathbf{x}_{2}\left(t_{0}\right)=\mathbf{0}$, so the results of the previous sections are valid for (3). Therefore, the derivation of a necessary condition for observability is ommitted, but a simpler sufficient condition can be found, as it will be detailed in the following subsections.

\section{E. Observability of the Linear System (second case)}

The following theorem provides a sufficient condition for the observability of (15).

Theorem 4: Suppose that the set of functions

$$
\mathcal{F}_{2}=\left\{u_{1}^{[1]}\left(t, t_{0}\right), u_{2}^{[1]}\left(t, t_{0}\right), u_{3}^{[1]}\left(t, t_{0}\right)\right\}
$$

is linearly independent on $\left[t_{0}, t_{f}\right], t_{0}<t_{f}$. Then, the LTVS (15) is observable on $\left[t_{0}, t_{f}\right]$ in the sense that, given the system input $\mathbf{u}(t), t \in\left[t_{0}, t_{f}\right]$, and the system output $y(t), t \in\left[t_{0}, t_{f}\right]$, the initial condition is uniquely defined.

Remark 1: The proofs for this theorem and the next follow the same reasoning as their counterparts in the first case, and were omitted due to space constraints.

\section{F. Observability of the Nonlinear System (second case)}

The following theorem provides a sufficient condition for the observability of the nonlinear system (3), as well as a practical result that can be used in the design of state observers for that system.

Theorem 5: Suppose that the set of functions (16) is linearly independent on $\left[t_{0}, t_{f}\right], t_{0}<t_{f}$. Then, the nonlinear system (3) is observable on $\left[t_{0}, t_{f}\right]$ in the sense that, given the system input $\mathbf{u}(t), t \in\left[t_{0}, t_{f}\right]$, and the system output $y(t), t \in\left[t_{0}, t_{f}\right]$, the initial condition is uniquely defined. Moreover, a state observer with globally asymptotically stable error dynamics for the LTVS (15) is also a state observer for the nonlinear system (3), with globally asymptotically stable error dynamics.

\section{Simulation Results}

This section provides simulation results that allow to assess the behavior and performance of the filtering solutions proposed in the paper. Due to lack of space, simulation results are only presented for (2).

Since (2) has an associated linear system which mimics its dynamics exactly, it is straightforward to design a globally asymptotically stable linear state observer [13]. In practice, one has to account for noise in the measurements, and also that it is the range that is usually measured, not its square, and squaring it may greatly amplify measurement noise. To address these issues, define a new augmented system state as

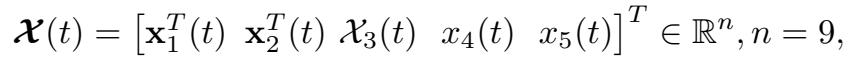

where $\mathcal{X}_{3}(t):=\left\|\mathbf{x}_{1}(t)\right\|=\sqrt{y(t)}$, and a new output as $z(t)=\left\|\mathbf{x}_{1}(t)\right\|$. Then, the new augmented system dynamics are

$$
\left\{\begin{array}{l}
\dot{\mathcal{X}}(t)=\mathbf{A}_{s}(t) \mathcal{X}(t)+\mathbf{B u}(t) \\
z(t)=\mathbf{C} \mathcal{X}(t)
\end{array}\right.
$$

where

$$
\mathbf{A}_{s}(t)=\left[\begin{array}{ccccc}
\mathbf{0} & \mathbf{I} & \mathbf{0} & \mathbf{0} & \mathbf{0} \\
\mathbf{0} & \mathbf{0} & \mathbf{0} & \mathbf{0} & \mathbf{0} \\
\frac{1}{z(t)} \mathbf{u}^{T}(t) & \mathbf{0} & 0 & \frac{1}{z(t)} & 0 \\
\mathbf{0} & \mathbf{u}^{T}(t) & 0 & 0 & 1 \\
\mathbf{0} & \mathbf{0} & 0 & 0 & 0
\end{array}\right], \mathbf{B}=\left[\begin{array}{c}
\mathbf{I} \\
\mathbf{0} \\
0 \\
0 \\
0
\end{array}\right]
$$

and $\mathbf{C}=\left[\begin{array}{lllll}\mathbf{0} & \mathbf{0} & 1 & 0 & 0\end{array}\right]$. Note that, assuming the output and its derivative are bounded for all time, which happens in practice, both augmented states are related through a Lyapunov coordinate transformation, thus preserving observability and stability properties [12]. Since (17) shares the observability properties of (4), it is straightforward to design a globally assymptotically stable Kalman filter for (17) that is robust to process and observation noise [14].

As for the parameters of the simulation, the source starts at $\mathbf{s}(0)=\left[\begin{array}{lll}0 & 0 & 0\end{array}\right]^{T}(\mathrm{~m})$, and its inertial velocity follows

$$
\mathbf{v}_{s}(t)=\left[\begin{array}{c}
1 \\
0.2 \cos \left(\frac{2 \pi}{200} t\right) \\
0
\end{array}\right] \quad(\mathrm{m} / \mathrm{s})
$$

The agent starts at $\mathbf{s}(0)=\left[\begin{array}{lll}0 & 0 & -10\end{array}\right]^{T}(\mathrm{~m})$, and is assumed to be moving in a fluid with velocity $\mathbf{v}_{f}=$ $\left[\begin{array}{lll}0.5 & -0.5 & -0.5\end{array}\right]^{T}(\mathrm{~m} / \mathrm{s})$ relative to $\{I\}$, expressed in inertial coordinates. The velocity of the agent relative to the fluid, also in inertial coordinates, follows

$$
\mathbf{v}_{r}(t)=\left[\begin{array}{c}
1.5+0.5 \cos \left(\frac{2 \pi}{10} t\right) \\
-0.5 \\
-0.5+0.5 \cos \left(\frac{2 \pi}{100} t\right)
\end{array}\right](\mathrm{m} / \mathrm{s}) .
$$

Note that the resulting system input, $\mathbf{v}_{s}(t)-\mathbf{v}_{r}(t)$, verifies the sufficient condition for observability expressed in Theorem 3. The measurement noise was simulated by adding a zero-mean, uncorrelated and normally distributed perturbation to the velocity and range measurements, with standard 

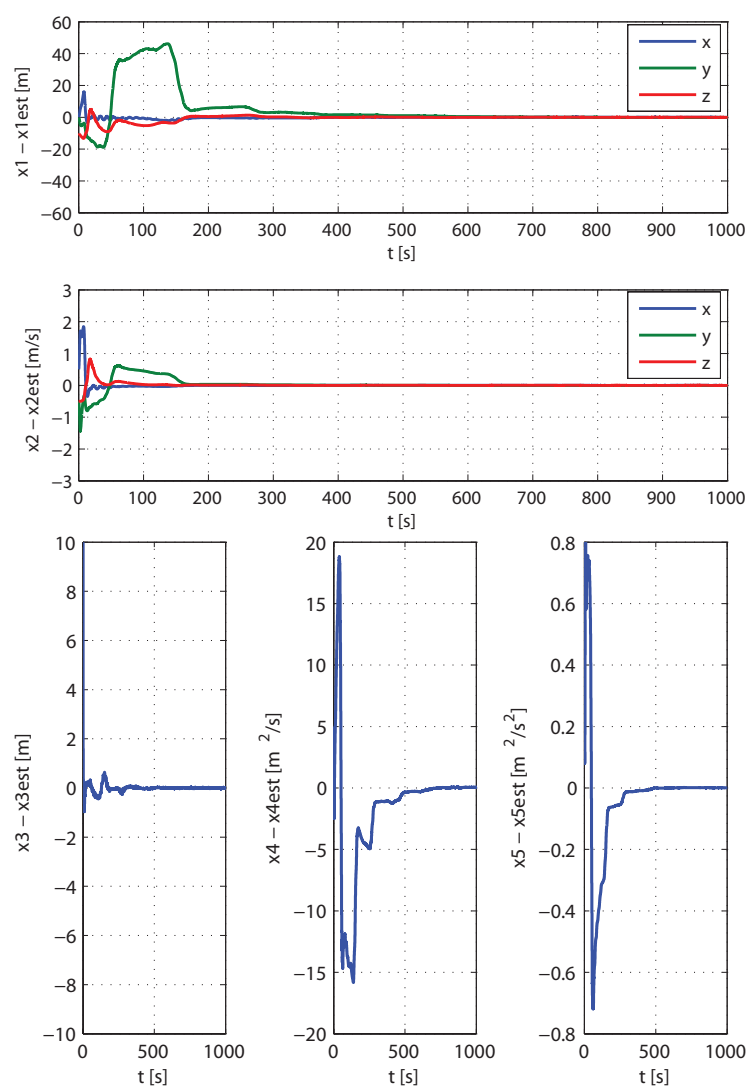

Fig. 1. Evolution of the estimation error variables

deviations of $0.01 \mathrm{~m} / \mathrm{s}$ and $0.2 \mathrm{~m}$, respectively. To tune the Kalman filter, the covariances were set to

$\left\{\begin{array}{l}\mathbf{Q}=0.01 \mathrm{diag}(1,1,1,0.001,0.001,0.001,1,1,0.001) \\ R=1\end{array}\right.$.

TABLE I

MEASURED STANDARD DEVIATION OF THE STEADY-STATE ESTIMATION ERROR OF RELEVANT VARIABLES

\begin{tabular}{l|c}
\hline Variable & Standard Deviation \\
\hline \hline $\mathbf{x}_{1}^{x}(t)$ & $2.73 \times 10^{-2}(\mathrm{~m})$ \\
$\mathbf{x}_{1}^{y}(t)$ & $3.65 \times 10^{-2}(\mathrm{~m})$ \\
$\mathbf{x}_{1}^{z}(t)$ & $1.28 \times 10^{-2}(\mathrm{~m})$ \\
$\mathbf{x}_{2}^{x}(t)$ & $2.78 \times 10^{-4}(\mathrm{~m} / \mathrm{s})$ \\
$\mathbf{x}_{2}^{y}(t)$ & $1.40 \times 10^{-4}(\mathrm{~m} / \mathrm{s})$ \\
$\mathbf{x}_{2}^{z}(t)$ & $1.39 \times 10^{-4}(\mathrm{~m} / \mathrm{s})$ \\
\hline
\end{tabular}

Fig. 1 details the evolution of the error variables. The large transients are caused by mismatches in the initial conditions. To complement the graphical data, Table I details the measured standard deviations of the steady-state estimation errors of the variables with the most practical interest, $\mathbf{x}_{1}$ and $\mathbf{x}_{2}$. As it can be seen, even under realistic sensor noise, the achieved values remain confined to small intervals and excellent filtering performance is achieved.

\section{COnClusions}

This paper presented a novel navigation solution for Autonomous Underwater Vehicles (AUVs) working in co- operation with an Autonomous Surface Craft (ASC), in which the AUV uses single range measurements as well as relevant information communicated by the ASC to estimate its own position. Nonlinear systems that model the dynamics of the two distinct cases were presented, and necessary and sufficient conditions for observability were derived. The solution presented here departs from classical solutions such as the Extended Kalman Filter (EKF), which relies on the linearization of the nonlinear system dynamics, by using state augmentation to derive linear systems which mimic the dynamics of the nonlinear systems exactly, allowing to guarantee global asymptotic stability. Although inspired in marine robotics, the proposed methods apply to other mobile platforms as they are based on the general motion kinematics in 3-D. Finally, to assess the performance of the proposed solutions, realistic simulation results were presented and discussed.

\section{REFERENCES}

[1] J. Vasconcelos, B. Cardeira, C. Silvestre, P. Oliveira, and P. Batista, "Discrete-Time Complementary Filters for Attitude and Position Estimation: Design, Analysis and Experimental Validation," IEEE Transactions on Control Systems Technology (accepted for publication), 2010.

[2] J. Kinsey, R. Eustice, and L. Whitcomb, "A Survey of Underwater Vehicle Navigation: Recent Advances and New Challenges," in Proceedings of the 7th IFAC Conference on Manoeuvring and Control of Marine Craft, Lisboa, Portugal, Sep. 2006.

[3] M. Kuritsky and M. Goldstein, Autonomous Robot Vehicles. SpringerVerlag, 1990.

[4] D. B. Heckman and R. C. Abbot, "An Acoustic Navigation Technique," in Proceedings of the Oceans MTS/IEEE, 1973, pp. 591-595.

[5] M. Hunt, W. Marquet, D. Moller, K. Peal, W. Smith, and R. Spindel, "An Acoustic Navigation System, Technical Report WHOI-74-6," Woods Hole Oceanographic Institution, Tech. Rep., 1974.

[6] P. H. Milne, Underwater Acoustic Positioning Systems. Houston, TX USA: Gulf Publishing Company, 1983.

[7] G. Antonelli, F. Arrichiello, S. Chiaverini, and G. Sukhatme, "Observability analysis of relative localization for AUVs based on ranging and depth measurements," in 2010 IEEE International Conference on Robotics and Automation, Anchorage, Alaska, USA, May 2010, pp. 4286-4271.

[8] A. Bahr, J. Leonard, and M. Fallon, "Cooperative localization for autonomous underwater vehicles," The International Journal of Robotics Research, 28, pp. 714-728, Jun. 2009.

[9] X. Zhou and S. Roumeliotis, "Robot-to-robot relative pose estimation from range," IEEE Transactions on Robotics, vol. 24, no. 6, pp. 13791393, Dec. 2008.

[10] A. Nehorai and E. Paldi, "Acoustic Vector-Sensor Array Processing," IEEE Transactions on Signal Processing, vol. 42, no. 9, pp. 24812491, Sep. 1994.

[11] P. Batista, C. Silvestre, and P. Oliveira, "Single Range Navigation in the presence of Constant Unknown Drifts," in Proceedings of the European Control Conference 2009, Budapest, Hungary, Aug. 2009, pp. 3983-3988.

[12] R. W. Brockett, Finite Dimensional Linear Systems. Wiley and Sons, 1970.

[13] W. J. Rugh, Linear System Theory, 2nd ed. Prentice Hall, 1996.

[14] A. H. Jazwinski, Stochastic Processes and Filtering Theory. Academic Press, 1970. 